\title{
Custos públicos atribuíveis a obesidade: amostra da USF Itamarati, Patos de Minas
}

\author{
Public costs attributable to obesity: sample from the USF Itamarati, Patos de Minas \\ Costos públicos atribuibles a la obesidad: muestra de la USF Itamarati, Patos de Minas
}

Recebido: 06/09/2021 | Revisado: 12/09/2021 | Aceito: 15/09/2021 | Publicado: 17/09/2021

\author{
Bárbara Queiroz de Figueiredo \\ ORCID: https://orcid.org/0000-0003-1630-4597 \\ Centro Universitário de Patos de Minas, Brasil \\ E-mail: barbarafigueiredo@unipam.edu.br \\ Caio Flávio Reis Nogueira \\ ORCID: https://orcid.org/0000-0003-1514-9660 \\ Centro Universitário de Patos de Minas, Brasil \\ E-mail: caioflavio@unipam.edu.br \\ Rúbia Carla Oliveira \\ ORCID: https://orcid.org/0000-0002-7162-3497 \\ Centro Universitário de Patos de Minas, Brasil \\ E-mail: rubiacoliveira@unipam.edu.br
}

\begin{abstract}
Resumo
Introdução: Quanto maior o índice de massa corporal (IMC) de um paciente, maior se torna o risco de o paciente desenvolver doenças relacionadas à obesidade. Da mesma forma, a gravidade da obesidade e doenças relacionadas aumenta à medida que o IMC aumenta. Objetivo: avaliar os custos da obesidade e doenças relacionadas no Brasil na perspectiva da sociedade e dos sistemas de saúde, perante análise de estimativa dos custos atribuíveis a essa patologia principal, considerando custos de procedimentos ambulatoriais, medicamentos distribuídos pelo SUS para tratamento da doença e solicitação de exame. Metodologia: abordagem observacional analítica desenvolvido na Unidade Básica de Saúde Itamarati, localizada no bairro Jardim Itamarati, na cidade de Patos de Minas, Minas Gerais, sob dados da Plataforma Viver, prontuários físicos e DATASUS. Resultados e discussão: perante resultados deste estudo e dos outros aqui citados, nota-se que, considerando separadamente a obesidade como fator de risco para hipertensão e diabetes, os custos atribuíveis a essa doença são altos, e as estimativas dos custos atribuíveis às principais doenças crônicas associadas à alimentação inadequada evidenciam a grande carga econômica dessas doenças para o SUS. Os dados mostram a necessidade de priorizar políticas integradas e intersetoriais para a prevenção e o controle da hipertensão, do diabetes e da obesidade. Conclusão: A obesidade impõe um custo alto à saúde das pessoas e da administração pública. Desse modo, ao compreender a fisiopatologia da obesidade, permitirá que os provedores em saúde gerenciem melhor o conjunto de doenças corroboradas por essa patologia principal que afetam um indivíduo.
\end{abstract}

Palavras-chave: Obesidade; Saúde pública; Custos; Gestão; Comorbidades.

\begin{abstract}
Introduction: The higher the body mass index (BMI) of a patient, the greater the risk of the patient developing obesity-related diseases. Likewise, the severity of obesity and related diseases increases as BMI increases. Objective: to assess the costs of obesity and related diseases in Brazil from the perspective of society and health systems, in view of the analysis of estimated costs attributable to this main pathology, considering the costs of outpatient procedures, medicines distributed by the SUS to treat the disease and request of exam. Methodology: analytical observational approach developed at the Itamarati Basic Health Unit, located in the Jardim Itamarati neighborhood, in the city of Patos de Minas, Minas Gerais, using data from the Viver Platform, physical records and DATASUS. Results and discussion: given the results of this study and others cited here, it is noted that, considering obesity as a risk factor for hypertension and diabetes separately, the costs attributable to this disease are high, and the estimates of costs attributable to the main diseases Chronic diseases associated with inadequate nutrition show the great economic burden of these diseases for the SUS. The data show the need to prioritize integrated and intersectoral policies for the prevention and control of hypertension, diabetes and obesity. Conclusion: Obesity imposes a high cost to the health of people and public administration. Thus, by understanding the pathophysiology of obesity, it will allow healthcare providers to better manage the set of diseases supported by this main pathology that affect an individual.
\end{abstract}

Keywords: Obesity; Public health; Costs; Management; Comorbidities. 


\begin{abstract}
Resumen
Introducción: Cuanto mayor es el índice de masa corporal (IMC) de un paciente, mayor es el riesgo de que el paciente desarrolle enfermedades relacionadas con la obesidad. Asimismo, la gravedad de la obesidad y las enfermedades relacionadas aumenta a medida que aumenta el IMC. Objetivo: evaluar los costos de la obesidad y enfermedades relacionadas en Brasil desde la perspectiva de la sociedad y los sistemas de salud, en vista del análisis de los costos estimados atribuibles a esta patología principal, considerando los costos de los procedimientos ambulatorios, medicamentos distribuidos por el SUS para tratar la enfermedad y solicitud de examen. Metodología: enfoque observacional analítico desarrollado en la Unidad Básica de Salud Itamarati, ubicada en el barrio Jardim Itamarati, en la ciudad de Patos de Minas, Minas Gerais, utilizando datos de la Plataforma Viver, registros físicos y DATASUS. Resultados y discusión: dados los resultados de este estudio y otros aquí citados, se observa que, considerando la obesidad como factor de riesgo de hipertensión y diabetes por separado, los costos atribuibles a esta enfermedad son elevados y las estimaciones de costos atribuibles a los principales Enfermedades Las enfermedades crónicas asociadas a una nutrición inadecuada muestran la gran carga económica de estas enfermedades para el SUS. Los datos muestran la necesidad de priorizar políticas integradas e intersectoriales para la prevención y control de la hipertensión, la diabetes y la obesidad. Conclusión: la obesidad impone un alto costo para la salud de las personas y la administración pública. Así, al comprender la fisiopatología de la obesidad, permitirá a los proveedores de salud manejar mejor el conjunto de enfermedades que sustenta esta patología principal que afecta a un individuo.
\end{abstract}

Palabras clave: Obesidad; Salud pública; Costos; Gestión; Comorbilidades.

\title{
1. Introdução
}

O maior mito na área da saúde é que a obesidade é uma problemática induzida, mas que pode ser curado comendo menos e realizando mais atividade física. Apesar de décadas de tratamento da obesidade com os tradicionais métodos de dieta e exercícios, pouco progresso foi feito. Embora esses métodos convencionais tenham sucesso nas pessoas que estão acima do peso, os pacientes com obesidade geralmente têm progressão além do ponto de inflexão, assim, as maneiras convencionais e fenotípicas de perder peso são menos eficazes. Estudos sugerem que a redefinição genética surge quando o tecido adiposo corrobora disfunção do tecido (Andolfi et al. 2018).

Quanto maior o índice de massa corporal (IMC) de um paciente, maior se torna o risco de o paciente desenvolver doenças relacionadas à obesidade. Da mesma forma, a gravidade da obesidade e doenças relacionadas aumenta à medida que o IMC aumenta. (Finkelstein et al., 2012). Triagem e medidas de avaliação do IMC de um paciente é fundamental, pois é uma ferramenta fundamental para identificar pacientes com IMC de $25 \mathrm{~kg} / \mathrm{m} 2$ e acima, que são classificados como com sobrepeso e estão em risco de progredir no quadro de obesidade e corroborar o aparecimento de doenças relacionadas. Pacientes com sobrepeso (IMC de 25-29,9 kg / m 2) geralmente ainda não apresentam ampliação avançada da redefinição genética, desse modo, mantê-los em faixa de sobrepeso ou trazê-los para um IMC mais baixo poderia prevenir doenças relacionadas à obesidade, haja vista que assim que um paciente ganha peso, o ambiente começa a impactar seus genes e mudar a maneira como os genes funcionam para controle de peso (Thorpe et al. 2015).

A resistência à insulina (RI) é um dos lados mais significativos efeitos da obesidade e foi reconhecida como característica integrante da síndrome metabólica, que inclui intolerância à glicose, hipertrigliceridemia, colesterol LDL alto, hipertensão e aterosclerose acelerada. Quando ocorre a RI, o pâncreas ainda pode produzir e secretar insulina, mas as célulasalvo são incapazes de responder efetivamente a concentrações sanguíneas. Isso, por sua vez, estimula as células beta no pâncreas para produzir níveis cada vez mais altos de insulina, tornando a hiperinsulinemia um dos principais sinais de desenvolvimento do diabetes mellitus (Pedersen et al. 2015).

A dislipidemia é um distúrbio do metabolismo das lipoproteínas em que existem quantidades anormais de lipídios no sangue, e é frequentemente encontrada em pacientes com obesidade e um dos primeiros sinais de que alguma disfunção metabólica está ocorrendo. Um em cada 3 americanos morre de doença cardíaca e acidente vascular cerebral, e ambas as doenças estão relacionadas à dislipidemia (Xu et al. 2015). À medida que a gordura abdominal aumenta, a gordura também se acumula em torno do coração. Ou seja, há evidências de que um aumento na gordura visceral possa produzir aumento de 
leptina e redução de adiponectina, e pode ser o sinal que acelera o acúmulo de placas ateroscleróticas. Aliado a isso, o diabetes mellitus aumenta o desenvolvimento de cardiomiopatia, levando a um quadro diabético distinto fenótipo miocárdico conhecido como cardiomiopatia diabética (Bueter et al. 2009).

A maioria da população brasileira depende exclusivamente do Sistema Único de Saúde (SUS) para obter assistência médica, enquanto apenas $25 \%$ da população geral tem acesso ao Sistema Suplementar de Saúde (aproximadamente 47 milhões de brasileiros). O financiamento desses sistemas é complexo e os recursos limitados. O crescente número de casos de obesidade configura, no longo prazo, uma ameaça à sustentabilidade do tratamento desses indivíduos tanto no âmbito público quanto no privado. Aliado a isso, é notório que a fisiopatologia da obesidade corrobora comorbidades posteriores, como a hipertensão arterial sistêmica (HAS), diabetes mellitus, distúrbios tireoidianos e dislipidemias (Oliveira et al. 2015). Aliado a isso, as doenças crônicas não transmissíveis (DCNTs) representam uma grande carga para o sistema público de saúde do Brasil, haja vista que são uma das principais causas de morte e de adoecimento da população, e, nesse sentido, devido seus efeitos negativos diretos na saúde, somados aos efeitos indiretos resultantes de doenças crônicas associadas, a obesidade representa uma carga dupla para os sistemas de saúde (Figueiredo et al. 2021).

Desse modo, o objetivo deste estudo é avaliar os custos da obesidade e doenças relacionadas no mundo e no Brasil na perspectiva da sociedade e dos sistemas de saúde, perante análise de estimativa dos custos atribuíveis a essa patologia principal, considerando custos de procedimentos ambulatoriais, medicamentos distribuídos pelo SUS para tratamento da doença e solicitação de exames, pode-se auxiliar a subsidiar o aprimoramento e a priorização das políticas de prevenção e enfrentamento das DCNTs, além de fortalecer a defesa de intervenções mais robustas em relação a medidas fiscais e regulatórias, aprimorando a gestão pública de saúde.

\section{Metodologia}

O presente estudo é pautado em uma abordagem observacional analítica desenvolvido na Unidade Básica de Saúde Itamarati, localizada no bairro Jardim Itamarati, na cidade de Patos de Minas, Minas Gerais, com o objetivo da criação de um perfil do paciente obeso da unidade, associando-os às suas respectivas comorbidades corroboradas pela doença principal, a fim de, posteriormente, calcular os custos do Sistema Único de Saúde (SUS) remanejados para esses pacientes, com base na Plataforma do SUS. A pesquisa foi realizada na USF Itamarati, por meio da Plataforma Viver e, posteriormente, por meio de análise individual e detalhada de prontuários físicos que estão presentes na Instituição. Os participantes incluídos no estudo foram todos aqueles cadastrados, sob atendimento na USF, com o CID E-66 (referente à obesidade) em seus prontuários, no período compreendido de um ano (03/03/20 a 03/03/21). Os pacientes cujo prontuários não estejam acessíveis, que não estejam residindo em localidades não atribuíveis a UBS Itamarati ou que faleceram foram excluídos desta amostra.

Para o seguinte estudo, coletou-se os números de prontuário eletrônico dos pacientes cujo CID E-66 estavam inclusos, no período correspondente de um ano. Após a coleta, foram avaliados, detalhadamente, os prontuários físicos dos respectivos pacientes. Posteriormente, foram calculados os custos atribuíveis a amostra, como atendimentos, medicações e exames, via tabulação de preços, de acordo Sistema de Gerenciamento da Tabela de Procedimentos, Medicamentos e OPM do SUS (DATASUS), de 2018. Este projeto foi submetido ao Comitê de Ética em Pesquisa do Centro Universitário de Patos de Minas via Plataforma Brasil. O mesmo obedecerá a legislação nacional vigente, para realização da pesquisa envolvendo seres humanos, conforme a Resolução do Conselho Nacional de Saúde 466/2012. O presente estudo contou com o aporte de trabalhos acadêmicos entre os anos de 2007 e 2021, por meio do acesso às principais bases de dados, a saber: dados National Library of Medicine (PubMed MEDLINE), Scientific Electronic Library Online (Scielo), Cochrane Database of Systematic Reviews (CDSR), Google Scholar, Biblioteca Virtual em Saúde (BVS) e EBSCO Information Services, no período compreendido entre abril e agosto de 2021. 


\section{Resultados e Discussão}

Para a disposição dos dados, foram avaliados, essencialmente, o número total de pacientes obesos cujo CID E-66 foi inserido no prontuário, e que estão atualmente cadastrados na unidade em questão. Conforme disposto na Tabela 1, a USF do bairro Itamarati abrange um total de 55 obesos cadastrados conforme CID E-66, sendo estes representados por 16 homens e 39 mulheres, respectivamente, $29 \%$ e $71 \%$, em que é possível observar uma prevalência geral de mulheres obesas. Além disso, conforme exposto na Tabela 2, a média de idade do perfil do paciente obeso é entre os 40 e 60 anos, o que ilustra a maior prevalência dessa patologia na idade adulta. Os dados referentes ao perfil do paciente obeso cadastrado atualmente na USF Itamarati, entre as idades 10-77 anos, foram coletados na Plataforma Viver, no período estipulado de 1 ano, e dispostos nas seguintes tabelas:

Tabela 1: Número total de pacientes da amostra divididos por sexo.

\begin{tabular}{|c|c|c|}
\hline Sexo & fa & fr (\%) \\
\hline Masculino & 16 & $29 \%$ \\
\hline Feminino & 39 & $71 \%$ \\
\hline Total & 55 & $100 \%$ \\
\hline
\end{tabular}

Fonte: Autores (2021).

Tabela 2: Número total de pacientes da amostra divididos por idade.

\begin{tabular}{|r|c|c|c|}
\hline \multicolumn{2}{|c|}{ Idade } & fa & fr (\%) \\
\hline 10 & ---20 & 4 & $7 \%$ \\
\hline 20 & ----30 & 4 & $7 \%$ \\
\hline 30 & ----40 & 11 & $20 \%$ \\
\hline 40 & ----50 & 13 & $24 \%$ \\
\hline 50 & ----60 & 14 & $26 \%$ \\
\hline 60 & ----70 & 6 & $11 \%$ \\
\hline 70 & ----80 & 3 & $5 \%$ \\
\hline \multicolumn{2}{|c|}{ Total } & 55 & $100 \%$ \\
\hline
\end{tabular}

Fonte: Autores (2021).

Partindo para análises específicas sobre as comorbidades possivelmente associadas e corroboradas pela obesidade, observa-se, pelo Gráfico 1, a prevalência de hipertensão arterial sistêmica (HAS), dislipidemias e hipotireoidismo, diabetes mellitus insulino-dependente e não dependente, sendo a HAS a patologia de maior associação, conforme análise singular de prontuários do espaço amostral. Outrossim, o Gráfico 2 expõe, de maneira quantitativa, o número de atendimentos ambulatoriais e de referenciações direcionados para essa amostragem. Em associação, no Gráfico 3 estão postulados o número total de exames solicitados para os pacientes estudados, sendo o hemograma total o mais requisitado pela atenção básica. 
Research, Society and Development, v. 10, n. 12, e179101220272, 2021

(CC BY 4.0) | ISSN 2525-3409 | DOI: http://dx.doi.org/10.33448/rsd-v10i12.20272

Gráfico 1: Associação quantitativa de pacientes obesos com comorbidades secundárias.

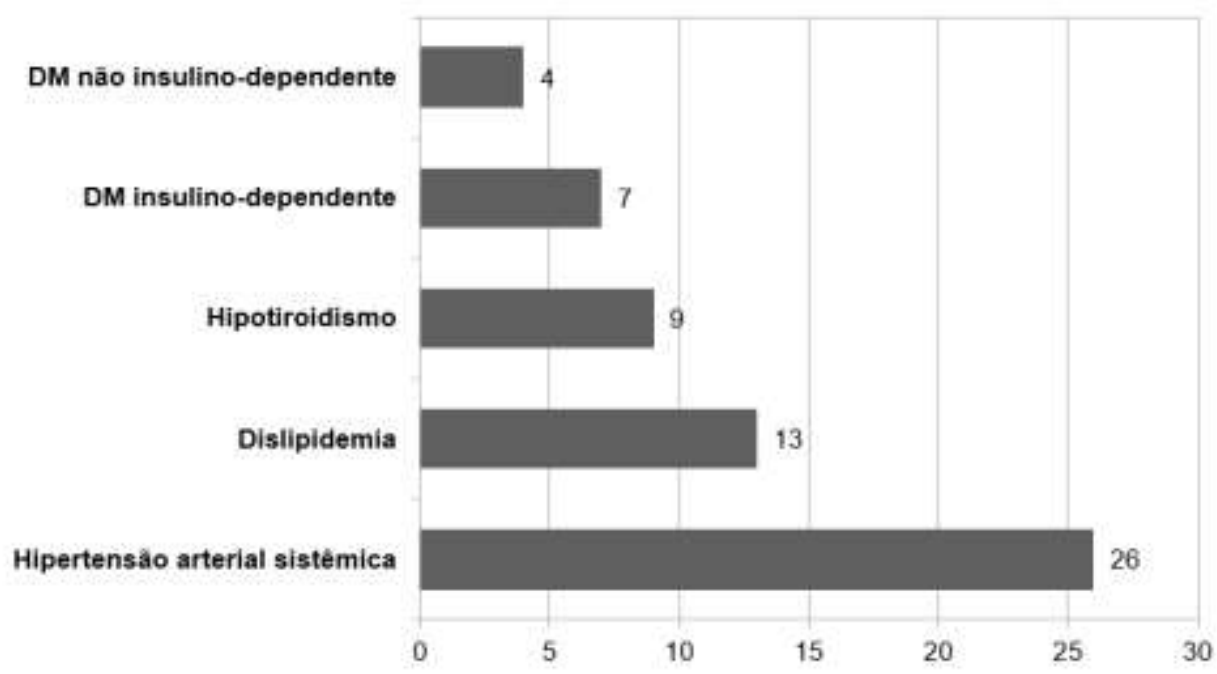

Fonte: Autores (2021).

Gráfico 2: Quantidade de atendimentos realizados à amostra.

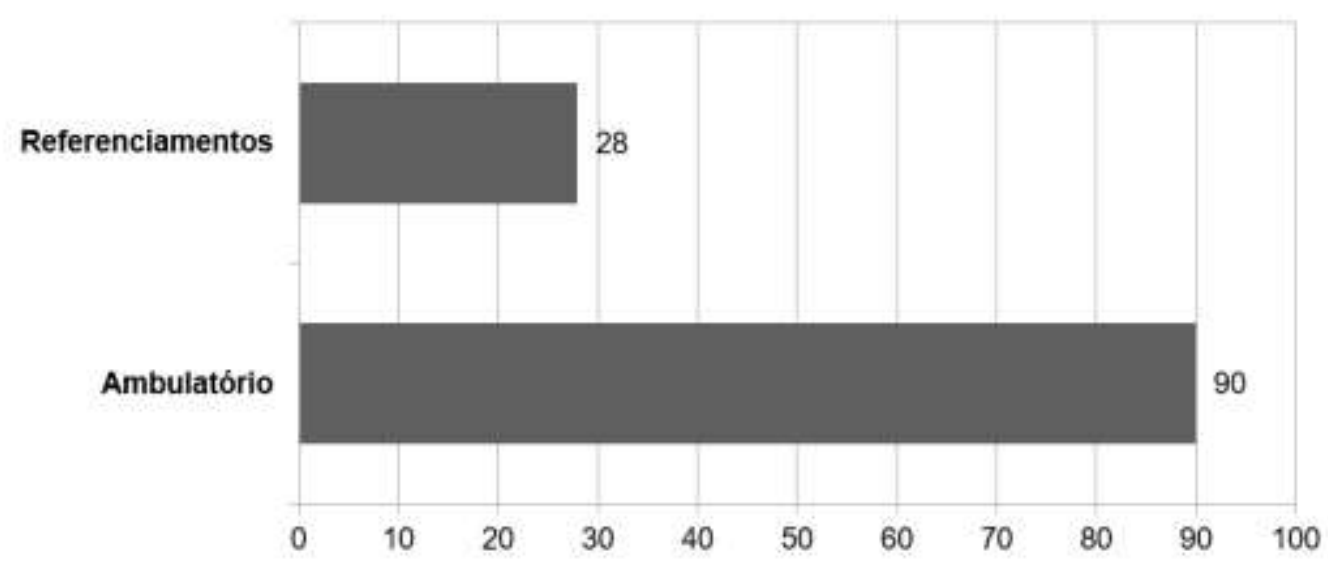

Fonte: Autores (2021).

Gráfico 3: Quantidade de exames complementares solicitados.

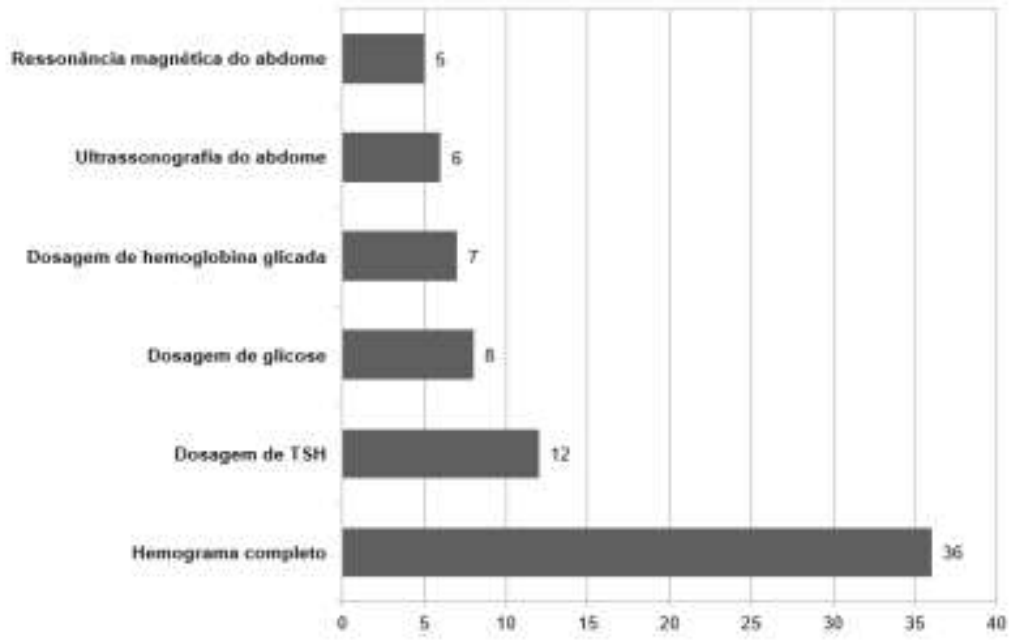

Fonte: Autores (2021). 
A tabulação dos custos dos referenciamentos, atendimentos ambulatoriais e exames complementares solicitados para a amostra deste estudo, estão descritas na Tabela 3 e Tabela 4, por meio do Sistema de Gerenciamento da Tabela de Procedimentos, Medicamentos e OPM do SUS (DATASUS), de 2018.

Tabela 3: Valores, em $\mathrm{R} \$$, dos atendimentos realizados para a amostra.

\begin{tabular}{|l|l|l|}
\hline Atendimentos & $\begin{array}{l}\text { Referenciamentos } \\
\text { especializadas) }\end{array}$ & $\begin{array}{l}\text { (consultas } \\
\text { Saúde }\end{array}$ \\
\hline Valor unitário & 56,00 & 60,60 \\
\hline Valor total & 1568,00 & 5454,00 \\
\hline
\end{tabular}

Fonte: Autores (2021).

Tabela 4: Valores, em $\mathrm{R} \$$, dos exames complementares solicitados para a amostra.

\begin{tabular}{|c|c|c|c|c|c|c|}
\hline $\begin{array}{c}\text { Exame } \\
\text { complementar }\end{array}$ & $\begin{array}{c}\text { Hemograma } \\
\text { completo }\end{array}$ & $\begin{array}{c}\text { Dosagem de } \\
\text { TSH }\end{array}$ & $\begin{array}{c}\text { Dosagem de } \\
\text { glicose }\end{array}$ & $\begin{array}{c}\text { Dosagem de } \\
\text { hemoglobina } \\
\text { glicosilada }\end{array}$ & $\begin{array}{c}\text { Ultrassonografia } \\
\text { de abdome }\end{array}$ & $\begin{array}{c}\text { Ressonância } \\
\text { magnética de } \\
\text { abdome }\end{array}$ \\
\hline Valor unitário & 4,11 & 8,96 & 1,85 & 7,86 & 68,40 & 268,75 \\
\hline Valor total & 147,96 & 107,52 & 14,80 & 55,02 & 410,40 & 1343,75 \\
\hline
\end{tabular}

Fonte: Autores (2021).

A seguir, na Tabela 5 estão elucidadas as medicações utilizadas pela amostragem, para tratamento de diabetes mellitus, dislipidemias, hipertensão arterial sistêmica e distúrbios tireoidianos, divididos por quantidade de pacientes que utilizam a medicação e número de caixas utilizadas durante o período de 12 meses (do estudo retrospectivo), e levando em consideração a dosagem de 1 comprimido ao dia das medicações orais, e de 10 UI/ML quando tratar-se de insulina humana injetável. Além disso, na tabela 6 estão elucidados os valores destes medicamentos, que foram estipulados por meio do Sistema de Gerenciamento da Tabela de Procedimentos, Medicamentos e OPM do SUS (DATASUS), de 2018.

Tabela 5: Análise quantitativa das medicações fornecidas pelo SUS para a amostra.

\begin{tabular}{|c|c|c|c|c|c|c|c|c|c|}
\hline Medicamento & $\begin{array}{l}\text { Losartana } \\
\text { Potássica } \\
\text { 50mg }\end{array}$ & $\begin{array}{l}\text { Captopril } \\
25 \mathrm{mg}\end{array}$ & $\begin{array}{l}\text { Hidroclorotiazida } \\
25 \mathrm{mg}\end{array}$ & $\begin{array}{l}\text { Atenolol } \\
25 \mathrm{mg}\end{array}$ & $\begin{array}{l}\text { Maleato de } \\
\text { enalapril 10mg }\end{array}$ & Sinvastatina $20 \mathrm{mg}$ & $\begin{array}{ll}\text { Cloridrato } & \mathrm{de} \\
\text { metformina } & \\
500 \mathrm{mg} & \\
\end{array}$ & $\begin{array}{l}\text { Insulina } \\
\text { Humana } \\
100 \mathrm{UI} / \mathrm{ML} \\
\end{array}$ & $\begin{array}{l}\text { Glibenclamida } \\
5 \mathrm{mg}\end{array}$ \\
\hline $\begin{array}{lr}\text { Quantidade } & \text { de } \\
\text { pacientes } & \text { que } \\
\text { utilizam } & \text { a } \\
\text { medicação } & \\
\end{array}$ & 21 & 3 & 15 & 2 & 3 & 10 & 5 & 3 & 1 \\
\hline 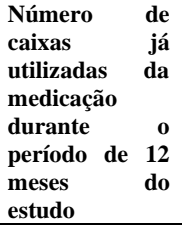 & 252 & 36 & 180 & 24 & 36 & 120 & 60 & 108 & 12 \\
\hline
\end{tabular}

Fonte: Autores (2021). 
Tabela 6: Valores, em R \$, de cada caixa de medicamento fornecido pelo SUS, em consonância à a Tabela 5.

\begin{tabular}{|c|c|c|c|c|c|c|c|c|c|}
\hline Medicamento & $\begin{array}{c}\text { Losartana } \\
\text { Potássica } \\
50 \mathrm{mg}\end{array}$ & $\begin{array}{l}\text { Captopril } \\
25 \mathrm{mg}\end{array}$ & $\begin{array}{c}\text { Hidroclorotiazida } \\
25 \mathrm{mg}\end{array}$ & $\begin{array}{l}\text { Atenolol } \\
25 \mathrm{mg}\end{array}$ & $\begin{array}{c}\text { Maleato de enalapril } \\
10 \mathrm{mg}\end{array}$ & $\begin{array}{l}\text { Sinvastatina } \\
\text { 20mg }\end{array}$ & $\begin{array}{l}\text { Cloridrato de } \\
\text { metformina } \\
500 \mathrm{mg}\end{array}$ & $\begin{array}{c}\text { Insulina } \\
\text { Humana } \\
100 \mathrm{UI} / \mathrm{ML}\end{array}$ & $\begin{array}{c}\text { Glibenclamida } \\
5 \mathrm{mg}\end{array}$ \\
\hline $\begin{array}{c}\text { Valor por } \\
\text { caixa }\end{array}$ & 4,80 & 2,70 & 1,50 & 3,00 & 4,50 & 6,90 & 6,90 & 20,08 & 2,10 \\
\hline Valor total & 1209,60 & 97,20 & 270,00 & 72,00 & 162,00 & 828,00 & 414,00 & 2168,64 & 25,20 \\
\hline
\end{tabular}

Fonte: Autores (2021).

Na Tabela 7, estão dispostos os custos totais de atendimentos (ambulatoriais e de referência), exames complementares e medicações, solicitados e ofertados para a amostra do estudo, bem como o gasto final.

Tabela 7: Valor total de gastos relacionados a obesidade.

\begin{tabular}{|l|l|l|l|}
\hline \multicolumn{1}{|c|}{ Atendimentos } & Exames complementares & Medicações & Total \\
\hline 7022,00 & 2079,45 & 5246,64 & $\mathbf{1 4 3 4 7 , 6 4}$ \\
\hline
\end{tabular}

Fonte: Autores (2021).

Estudo de Sichieri et al. (2007) estimou os custos de hospitalizações relacionados às doenças associadas ao sobrepeso/obesidade através dos dados das hospitalizações de homens e mulheres de 20 a 60 anos do Sistema de Informações Hospitalares do Sistema Único de Saúde (SIH-SUS) para o ano de 2001. Demonstraram que cerca de 3 a $5 \%$ de todas as internações no Brasil foram decorrentes de doenças relacionadas diretamente à obesidade. Além disso, avaliaram também dados de perda de produtividade pela identificação do número de dias de hospitalização. O número de dias de trabalho perdidos em função da obesidade e doenças associadas foi de 3,9 a 10,6 dias/ano. A causa mais comum de perda de produtividade foi o diabetes mellitus, seguido pelas doenças cardiovasculares (Hammond et al., 2010).

As doenças cardiovasculares, provavelmente pela maior frequência, foram responsáveis por $67 \%$ dos custos, seguida pelo tratamento do câncer. Considerando que os custos indiretos (perda de produtividade, licenças médicas, morte prematura) não foram incluídos e os dados de gastos médicos foram obtidos através do banco de dados do DATASUS, ou seja, valores reembolsados às unidades de saúde, os autores ressaltam que esses números são uma estimativa bastante conservadora dos gastos públicos com pacientes obesos, já que o custo real do tratamento é classicamente maior do que os valores reembolsados. Os custos estimados foram equivalentes a 0,09\% do PIB nacional em 2010, muito abaixo dos demonstrados em estudos internacionais (Allender et al., 2007; IBGE, 2010).

Bahia et al. (2012) demonstraram os custos médicos relacionados ao tratamento ambulatorial e hospitalar de doenças associadas ao sobrepeso e à obesidade nos anos de 2008 a 2010. Com base nos riscos relativos (dados internacionais) e prevalência de sobrepeso e obesidade no Brasil pode-se estimar a fração atribuível da obesidade sobre desfechos de interesse. Estimou-se que o SUS gasta anualmente cerca de R\$ 3,6 bilhões por ano com o tratamento dessas doenças, sendo R\$ 2,4 bilhões com o tratamento hospitalar (68\%) e R $\$ 1,2$ bilhões (32\%) com o tratamento ambulatorial, conforme demonstrado na tabela 8 . 
Tabela 8: Custos do SUS com o tratamento ambulatorial e hospitalar com as doenças relacionadas à obesidade (média dos anos de 2008 a 2010).

\begin{tabular}{|c|c|c|c|c|}
\hline Grupo de doenças & & $\begin{array}{c}\text { Custos ambulatoriais } \\
(\mathbf{R} \$)\end{array}$ & $\begin{array}{c}\text { Custos } \\
\text { hospitalares }(\mathbf{R} \$)\end{array}$ & Custos totais (R\$) \\
\hline & $\begin{array}{l}\text { Cardiovascular } \\
\text { (todas) }\end{array}$ & 148.593 .269 & 1.128 .947 .735 & 1.277 .541 .004 \\
\hline & $\begin{array}{c}\text { Doença arterial } \\
\text { coronariana }\end{array}$ & 110.805 .178 & 662.593 .988 & 773.399 .166 \\
\hline \multirow[t]{3}{*}{ Cardiovascular } & $\begin{array}{l}\text { Insuficiência } \\
\text { cardíaca }\end{array}$ & 2.592 .687 & 269.357.625 & 271.950 .312 \\
\hline & Hipertensão arterial & 23.103 .255 & 37.287 .453 & 60.390 .708 \\
\hline & $\begin{array}{l}\text { Acidente vascular } \\
\text { cerebral }\end{array}$ & 12.092 .150 & 159.708 .669 & 171.800 .819 \\
\hline Neoplasias* & & 407.377 .251 & 102.321 .674 & 509.698 .926 \\
\hline Diabetes mellitus** & & 1.447 .915 & 37.212 .371 & 40.376 .996 \\
\hline Osteoartrite*** & & 6.696 .476 & 9.907 .512 & 16.603 .988 \\
\hline
\end{tabular}

*relacionadas ao sobrepeso (colorretal, endométrio, ovário, pâncreas, mama, rim, bexiga); **apenas como primeiro diagnóstico; ***apenas joelho e quadril.

Fonte: Bahia et al. 2014.

Desse modo, perante resultados deste estudo e dos outros aqui citados, nota-se que, considerando separadamente a obesidade como fator de risco para hipertensão e diabetes, os custos atribuíveis a essa doença são altos, e as estimativas dos custos atribuíveis às principais doenças crônicas associadas à alimentação inadequada evidenciam a grande carga econômica dessas doenças para o SUS. Os dados mostram a necessidade de priorizar políticas integradas e intersetoriais para a prevenção e o controle da hipertensão, do diabetes e da obesidade.

\section{Conclusão}

A obesidade impõe um custo alto à saúde das pessoas e da administração pública. Desse modo, ao compreender a fisiopatologia da obesidade, permitirá que os provedores em saúde gerenciem melhor o conjunto de doenças corroboradas por essa patologia principal que afetam um indivíduo. Ademais, mantê-la como o foco central e base de todas as estratégias de tratamento para a obesidade e comorbidades relacionadas é essencial e de suma importância para a gestão de doenças atuais, bem como a prevenção de doenças futuras.

Dessa maneira, o conhecimento acerca dos custos atribuíveis a doenças pode subsidiar o aprimoramento e a priorização das políticas de prevenção e enfrentamento das DCNTs, além de fortalecer a defesa de intervenções mais robustas para prevenção dessas doenças, inclusive medidas fiscais e regulatórias, assim, agregar a obesidade a essas comorbidades permite uma estimativa mais completa do impacto econômico da obesidade no SUS (Figueiredo et al. 2021). Portanto, é de suma importância que estudos posteriores abranjam a temática, principalmente com o intuito de aumentar a sensibilidade para a gestão pública, revelar as áreas com maiores despesas e ajudar na identificação das áreas que requerem mais intervenções e medidas preventivas contra a obesidade. 
Research, Society and Development, v. 10, n. 12, e179101220272, 2021

(CC BY 4.0) | ISSN 2525-3409 | DOI: http://dx.doi.org/10.33448/rsd-v10i12.20272

\section{Referências}

Allender, S. \& Rayner, M. (2007). The burden of overweight and obesity-related ill health in the UK. Obes Rev. 8(5), 467-473.

Andolfi, C. \& Fisichella, P. M (2018). Epidemiologia da obesidade e comorbidades associadas. Revista de Técnicas Cirúrgicas e Laparoendoscópicas Avançadas, 28 (8), 1-6.

Bahia, L. R. \& Araújo, D. V. (2014). Impacto econômico da obesidade no Brasil. Revista HUPE, 13 (1), 13-17.

Brasil, Ministério da Saúde (2018). Banco de dados do Sistema Único de Saúde (DATASUS). http://sigtap.datasus.gov.br/tabela-unificada/app/sec/inicio.jsp.

Bueter, M., Ahmed, A. \& Ashrafian, H. (2009). Cirurgia bariátrica e hipertensão. Surg Obes Relat Dis, 5 (1), 615-620

Figueiredo, B. Q., Souto, B. O. V., Nogueira, C. F. R., Silva, I. T., Bernardes, L. B. R., Peres, M. L. A. \& Oliveira, R. C. (2021). O enorme custo da obesidade para a saúde pública brasileira: Uma breve revisão de literatura. Research, Society and Development. 10 (9), 1-9. http://dx.doi.org/10.33448/rsd-v10i9.18276

Finkelstein, E. A., Khavjou, O. A., Thompson, H., Trogdon, J. G., Pan, L. \& Sherry B. (2012). A obesidade e as previsões de obesidade grave até 2030. Am J Prev Med, 42 (1), 563-570

Hammond, R. A. \& Levine, R. (2010). The economic impact of obesity in the United States. Diabetes Metab Syndr Obes. 3 (1), $285-295$.

http://dx.doi.org/10.2147/DMSOTT.S7384

Instituto Brasileiro de Geografia e Estatística. Produto Interno Bruto (2010). IBGE.: http://www.ibge.gov.br/home/

Oliveira, M. L. (2013). Estimativa dos custos da obesidade para o Sistema Único de Saúde do Brasil [Tese de Doutorado em Nutrição Humana]. Brasília: Universidade de Brasília.

Pedersen, D. J., Guilherme, A., Danai, L. V., Heyda, L., Mate-vossian, A. \& Cohen J. (2015). Um importante papel da insulina no pro- inflamação do tecido adiposo associada à obesidade. Mol Metab, 4 (1), 507-518

Sichieri, R., Nascimento, S. \& Coutinho, W. (2007). The burden of hospitalization due to overweight and obesity in Brazil. Cad Saúde Pública. 23 (7), 17211727. http://dx.doi.org/10.1590/S0102-311X2007000700025

Thorpe, R. J., Júnior, K. E., Bowie, J. V., Griffith, D. M., Bruce, M. \& LaVeist, T. (2015). Explicando as disparidades raciais na obesidade entre homens: O lugar importa? Am J Mens Health, 9 (1), 464-472

Xu, W., Shubina, M., Goldberg, S. I. \& Turchin, A. (2015). Massa corporal índice e mortalidade por todas as causas em pacientes com hipertensão. Obesity, $23(1), 1712-1720$ 